PROCEEDINGS OF THE

AMERICAN MATHEMATICAL SOCIETY

Volume 124, Number 1, January 1996

\title{
WEIGHTED INEQUALITIES FOR THE MAXIMAL GEOMETRIC MEAN OPERATOR
}

\author{
XIANGRONG YIN AND BENJAMIN MUCKENHOUPT
}

(Communicated by J. Marshall Ash)

\begin{abstract}
For nonnegative Borel measures $\mu$ on $R^{1}$ and for the maximal geometric mean operator $G_{f}$, we characterize the weight pairs $(w, v)$ for which $G_{f}$ is of weak type $(p, p)$ and of strong type $(p, p), 0<p<\infty$. No doubling conditions are needed. We also note that a previously published different characterization for the strong type inequality for $G_{f}$ has an incorrect proof.
\end{abstract}

\section{INTRODUCTION}

Let $\mu$ be a nonnegative Borel measure on $R^{1}$. The maximal geometric mean operator is (see X. Shi [7])

$$
G_{f}(x)=\sup \exp \left[\frac{1}{|I|} \int_{I} \log (|f(y)|) d \mu(y)\right],
$$

where the sup is taken over all intervals $I$ in $R^{1}$ containing $x$ such that the integral is defined and $0<|I|=\mu(I)<\infty$; if no such $I$ exists we take $G_{f}(x)=0$.

If $0<p<\infty, w=v$ and $d \mu$ is Lebesgue measure on $R^{1}$, then the inequality

$$
\int G_{f}(x)^{p} w(x) d \mu(x) \leq C_{1} \int|f(x)|^{p} v(x) d \mu(x)
$$

for all $f$ in $L^{p}(v d \mu)$ is equivalent to the requirement that

$$
\left(\frac{1}{|I|} \int_{I} w d \mu\right)\left(\exp \left[\frac{1}{|I|} \int_{I} \log (1 / v) d \mu\right]\right) \leq C_{2}
$$

for all intervals $I$ (see [7]) and is also equivalent to the weak type inequality

$$
\int_{\left\{x \mid G_{f}(x)>\lambda\right\}} w d \mu \leq \frac{C_{3}}{\lambda^{p}} \int|f(x)|^{p} v(x) d \mu(x)
$$

for all $f$ in $L^{p}(v d \mu)$ (see [3]). In [3] it is stated that in spaces of homogeneous type condition (2) implies inequality (1) for general not necessarily equal $v$ and $w$ provided that $\mu$ and $v d \mu$ satisfy doubling conditions. The proof given in [3], however, is incorrect. They state on page 71 that "it is easy to see that" if $f_{0}(x)=$ $f(x)$ on the set where $|f(x)| \leq \alpha / 2$ and 0 elsewhere and $f_{1}(x)=f(x)-f_{0}(x)$, then $G_{f}(x) \leq G_{f_{1}}(x)+\alpha / 2$. A simple counterexample is $f(x)=\chi_{(0,1]}(x, x)+$ $e^{n} \chi_{(1, \infty)}(x, x)$ and $\alpha=2$; for $0<x<1$ we have $G_{f}(x)=e^{n}$ and $G_{f_{1}}(x)=0$. We

Received by the editors January 26, 1993 and, in revised form, January 31, 1994 and May 18, 1994.

1991 Mathematics Subject Classification. Primary 26D15, 42B25.

(C)1996 American Mathematical Society 
will show in Theorem 1 that in general a stronger condition than (2) is needed to imply (1).

It might seem that since condition (2) implies (3) for all $p$, the Marcinkiewicz interpolation theorem would show that (2) implies (1). The Marcinkiewicz theorem cannot be applied here, however, because the operator is not quasilinear. This is easily seen in $R^{1}$ with $d \mu=d x$ by taking $E$ to be a set such that $E \cap I$ and $E^{c} \cap I$ both have positive measure for every interval $I$. Then with $f=\chi_{E}$ and $g=\chi_{E^{c}}$ we have $G_{f+g}$ equal to 1 for all $x$ while $G_{f}$ and $G_{g}$ are 0 for all $x$.

\section{The Main theOREMS}

Theorem 1. Let $\mu$ be a nonnegative Borel measure on $R^{1}, 0<p<\infty$, and let $w$ and $v$ be two $\mu$ measurable weight functions. Then inequality (1) holds for all $f$ in $L^{p}(v d \mu)$ if and only if

$$
\int_{I} G_{\left(v^{-1} \chi_{I}\right)}(x) w(x) d \mu(x) \leq C_{4}|I|
$$

for every interval $I$.

Theorem 2. Let $w, v, \mu$ and $p$ be as in Theorem 1. Then inequality (3) holds for all $f$ in $L^{p}(v d \mu)$ if and only if (2) holds for every interval $I$.

From the proof of X. Shi [7] we can easily see that (4) is equivalent to (2) if $w=v$. This is also a simple consequence of Theorem 1 and well-known facts by the following reasoning. If $w=v$ satisfies (2), then by Theorem 1, page 254 of [2], $w$ satisfies the condition $A_{\infty}$. By the Theorem on page 104 of [4] there is a $p>1$ such that $w$ satisfies $A_{p}$, and since $G_{f}(x) \leq M f(x)$, where $M$ denotes the Hardy-Littlewood maximal operator, we have (1) by Theorem 2, page 216 of [5]. Theorem 1 then shows that $w$ satisfies (4).

Without the assumption $w=v$ conditions (2) and (4) are not equivalent; in section 6 we give an example of a pair of functions $v, w$ that satisfy (2) but do not satisfy (4).

These theorems can be viewed as limiting cases of known results about the Hardy-Littlewood maximal operator $M$. This is based on the equality

$$
\lim _{p \rightarrow \infty}\left(\frac{1}{|I|} \int_{I} v^{-1 / p} d \mu\right)^{p}=\exp \left[\frac{1}{|I|} \int_{I} \log (1 / v) d \mu\right],
$$

which is valid if the left side is finite (see [1], [2]), and the consequence that $G_{f}(x)=$ $\lim _{p \rightarrow \infty}\left[M\left(f^{1 / p}\right)\right]^{p}$ for suitably restricted $f$. Theorem 1 is a limiting case of the fact from Theorem B, p. 7 of [6] that $\int\left[M\left(f^{1 / p}\right)\right]^{p} w d \mu \leq C \int|f| v d \mu$ if and only if for every interval $I \int_{I}\left[M\left(\chi_{I} v^{-1 /(p-1)}\right)\right]^{p} w d \mu \leq C \int_{I} v^{-1 /(p-1)} d \mu$. Theorem 2 is a limiting case of the fact from Theorem 6, p. 219 of [5] that

$$
\int_{\left\{x \mid\left[M\left(f^{1 / p}\right)\right]^{p}>\lambda\right\}} w d \mu \leq \frac{C}{\lambda} \int|f| v d \mu
$$

if and only if for every interval $I$

$$
\left(\frac{1}{|I|} \int_{I} w d \mu\right)\left(\frac{1}{|I|} \int_{I} v^{-1 /(p-1)} d \mu\right)^{p-1} \leq C
$$




\section{COVERING LEMMAS}

Our proofs of the theorems are based on the following lemmas. Lemma 1 is the covering idea used in the proof of Theorem (a), p. 1231 of [8].

Lemma 1. If $\nu$ is a Borel measure, $E$ is a subset of $R^{1}, B$ is a collection of intervals that cover $E$ and there is a constant $C$ such that $0<\nu(I) \leq C$ for every $I$ in $B$, then there is a disjoint sequence $\left\{I_{j}\right\}$ of intervals in $B$ such that $\nu(E) \leq \sum 5 \nu\left(I_{j}\right)$.

To prove this each $I_{j}$ is chosen to be disjoint from $I_{1}, \ldots, I_{j-1}$ and with $\nu\left(I_{j}\right)>$ $(1 / 2) \sup \nu(I)$, where the sup is taken over all $I$ 's in $B$ disjoint from $I_{1}, \ldots, I_{j-1}$. If $\sum \nu\left(I_{j}\right)=\infty$, there is nothing more to prove. Therefore, assume that $\sum \nu\left(I_{j}\right)<\infty$. For each $I_{j}$ define $I_{j}^{*}$ to be the union of all intervals $H$ in $B$ that intersect $I_{j}$ and have $\nu(H)<2 \nu\left(I_{j}\right)$. Then $\nu\left(I_{j}^{*}\right) \leq 5 \nu\left(I_{j}\right)$ is immediate. Furthermore, if $x$ is in $E$, let $J$ be a member of $B$ that contains $x$. Since $\lim _{j \rightarrow \infty} \nu\left(I_{j}\right)=0$, there is a first member $I_{j}$ of the sequence that intersects $J$. Then by the selection procedure $\nu\left(I_{j}\right)>(1 / 2) \nu(J)$ and by its definition $J \subset I_{j}^{*}$. From this $E \subset \bigcup I_{j}^{*}$. Therefore, $\nu(E) \leq \sum \nu\left(I_{j}^{*}\right) \leq \sum 5 \nu\left(I_{j}\right)$. This completes the proof of Lemma 1.

Lemma 2. If $E$ is a subset of $R^{1}$ and $B$ is a collection of closed intervals of positive length that cover $E$, then $E$ is covered by a countable subcollection of $B$.

To prove this let $A$ be the set of points in $E$ that occur only as left ends of intervals in $B$. For every $x$ in $A$ there is an interval in $B$ that contains it, and this interval contains no other points of $A$ in its interior. Therefore, $A$ is countable and is consequently covered by a countable subcollection from $B$. The same reasoning applies to the points that occur only as right end points. The other points of $E$ are covered by the open interiors of the intervals in $B$, and there is a countable subcollection that covers them by Lindelöf's theorem.

\section{Proof of Theorem 1}

The proof that (1) implies (4) is trivial by taking $f(x)=v(x)^{-1 / p} \chi_{I}(x)$.

For the proof that (4) implies (1) we need only consider the case $p=2$ since $G_{f}(x)^{p}=G_{|f|^{p}}(x)$. It is enough to prove the result for bounded functions $f$ with support $E$ having finite $\mu$ measure and $\int|f|^{2} v d \mu<\infty$. For these $f$ 's it is enough to consider $v$ 's with positive lower bound by the following reasoning. Given arbitrary $v(x)$, let $v_{n}(x)$ be the larger of $1 / n$ and $v(x)$. Then $v_{n}$ will still satisfy (4) with the same value of $C_{4}$. It will appear in the proof that the constant $C_{1}$ depends only on $C_{4}$. Therefore, (1) will be valid for all $v_{n}$ 's with fixed $C_{1}$, and the restriction on $f$ insures that the right side of (1) converges properly as $n \rightarrow \infty$.

Having fixed such an $f$ and $v$, we define a function $F$ on intervals $I$ in $R^{1}$ by

$$
F(I)=\exp \left[\frac{1}{|I|} \int_{I} \log (|f(y)|) d \mu(y)\right]
$$

for intervals with $0<|I|<\infty$ for which the integral is defined and $F(I)=0$ on other intervals. For each $x$ for which $G_{f}(x)>0$ we will now construct a closed interval $I_{x}$ containing $x$ such that $F\left(I_{x}\right)>G_{f}(x) / 2$ and so that the end points of $I_{x}$ are measurable functions of $x$.

To do this let $E_{k}$ be the set of $x$ for which $2^{k}<G_{f}(x) \leq 2^{k+1}$, and let $C_{k}$ consist of all closed intervals $I$ with $2^{k}<F(I) \leq 2^{k+1}$. Let $A$ be the points $a$ in $E_{k}$ with $[a, a] \in C_{k}$. The set $A$ is countable since if $a$ is in $A,|[a, a]|>0$ and $a$ lies in the 
support of $f$ which has been assumed to have finite measure. Therefore, there is a countable subcollection of $C_{k}$ that covers $A$. If $x \in E_{k} \cap A^{c}$ there is an interval $I$ containing $x$ with $F(I)>2^{k}$ and $I$ must have positive length since $[x, x]$ is not in $C_{k}$. If one or both ends of $I$ are open, a closed interval $J$ of positive length can be found inside $I$ that contains $x$ and has $F(J)>2^{k}$. Therefore, the intervals of positive length in $C_{k}$ cover $E_{k} \cap A^{c}$, and by Lemma 2 there is a countable subcollection that covers this set. Let $\left\{J_{n}\right\}$ be a sequence of closed intervals in $C_{k}$ that cover $E_{k}$. For each $x$ in $E_{k}$ define $I_{x}$ to be the first $J_{n}$ that contains $x$. Then for $x$ in $E_{k}$ we have $F\left(I_{x}\right)>2^{k} \geq G_{f}(x) / 2$ and the ends of $I_{x}$ are measurable functions because the set where $I_{x}=J_{n}$ is the measurable set $J_{n} \cap E_{k} \cap \bigcap_{m=1}^{n-1} J_{m}^{c}$.

Then if $B$ is the set where $G_{f}(x)>0$,

$$
\begin{aligned}
\int G_{f}(x)^{2} w(x) d \mu(x)< & \int_{B} 4\left[\exp \left(\frac{1}{\left|I_{x}\right|} \int_{I_{x}} \log (|f|) d \mu\right)\right]^{2} w(x) d \mu(x) \\
= & 4 \int_{B}\left[\exp \left(\frac{1}{\left|I_{x}\right|} \int_{I_{x}} \log \left(|f| v^{1 / 2}\right) d \mu\right)\right]^{2} \\
& \times \exp \left(\frac{1}{\left|I_{x}\right|} \int_{I_{x}} \log \left(\frac{1}{v}\right) d \mu\right) w(x) d \mu(x) .
\end{aligned}
$$

Some justification is needed for this equality since in general an equality of the form

$$
\exp \left[\int_{I} \log (g) d \mu\right]=\exp \left[\int_{I} \log (g h) d \mu\right] \exp \left[\int_{I} \log (1 / h) d \mu\right]
$$

can fail if the right side has the form $0 \cdot \infty$. This does not happen here because the restrictions on $f$ and $v$ insure that $\int_{I_{x}} \log (|f|) d \mu, \int_{I_{x}} \log \left(|f| v^{1 / 2}\right) d \mu$ and $\int_{I_{x}} \log (1 / v) d \mu$ are all finite. By Jensen's inequality (5) is bounded by

$$
4 \int_{B}\left[\frac{1}{\left|I_{x}\right|} \int_{I_{x}}|f| v^{1 / 2} d \mu\right]^{2} \exp \left[\frac{1}{\left|I_{x}\right|} \int_{I_{x}} \log \left(\frac{1}{v}\right) d \mu\right] w(x) d \mu(x) .
$$

Define the measure $U$ by $d U(x)=w(x) \exp \left(\frac{1}{\left|I_{x}\right|} \int_{I_{x}} \log \left(\frac{1}{v}\right) d \mu\right) d \mu(x)$, and let $T$ be the linear operator defined by $T g(x)=\frac{1}{\left|I_{x}\right|} \int_{I_{x}} g d \mu$. Then $T$ is clearly bounded from $L^{\infty}(d \mu)$ to $L^{\infty}(d U)$. We shall show that $T$ is bounded from $L^{1}(d \mu)$ to weak $L^{1}(d U)$. This will be sufficient since these imply that $T$ is bounded from $L^{2}(d \mu)$ to $L^{2}(d U)$ and, therefore, that (6) equals

$$
4 \int_{B} T\left(f v^{1 / 2}\right)^{2} d U \leq C \int\left|f v^{1 / 2}\right|^{2} d \mu=C \int|f|^{2} v d \mu .
$$

To prove the weak type assertion for $T$ fix $g$ in $L^{1}(d \mu)$ and $\lambda>0$, let $D=$ $\{T g(x)>\lambda\}$, and let $E=\bigcup_{x \in D} I_{x}$. Since the set of all $I_{x}$ 's is countable, $E$ can be written as the countable union of its connected components $\left\{J_{n}\right\}$. Note that if $x$ is in $D \cap J_{n}$, then $I_{x} \subset J_{n}$. We have, therefore,

$$
\begin{aligned}
\int_{D} d U & =\sum_{n} \int_{D \cap J_{n}} \exp \left[\frac{1}{\left|I_{x}\right|} \int_{I_{x}} \log \left(\left|\frac{1}{v}\right|\right) d \mu\right] w(x) d \mu(x) \\
& \leq \sum_{n} \int_{J_{n}} \exp \left[\frac{1}{\left|I_{x}\right|} \int_{I_{x}} \log \left(\left|\frac{1}{v}\right| \chi_{J_{n}}\right) d \mu\right] w(x) d \mu(x) .
\end{aligned}
$$


By condition (4) this is bounded by

$$
C_{4} \sum_{n}\left|J_{n}\right| \cdot
$$

Now each $J_{n}$ is the union of $I_{x}$ 's with $x$ in $D$. From the fact that $x$ is in $D$ we have $0<\left|I_{x}\right|<\frac{1}{\lambda} \int_{I_{x}} g d \mu \leq \frac{1}{\lambda}\|g\|_{1}$. We can, therefore, apply Lemma 1 to each $J_{n}$. This will produce disjoint sequences of intervals $I_{n, k} \subset J_{n}$, and since the $I_{n, k}$ 's are $I_{x}$ 's with $x \in D$,

$$
\lambda<\frac{1}{\left|I_{n, k}\right|} \int_{I_{n, k}}|g| d \mu .
$$

Furthermore, since the $J_{n}$ 's are disjoint, all the intervals $I_{n, k}$ are disjoint. Using these two facts and Lemma 1 shows that (7) has the bound

$$
5 C_{4} \sum_{n, k}\left|I_{n, k}\right|<\sum_{n, k} \frac{5}{\lambda} C_{4} \int_{I_{n, k}}|g| d \mu \leq \frac{5}{\lambda} C_{4}\|g\|_{1} .
$$

This completes the proof of Theorem 1.

\section{Proof of Theorem 2}

The proof that (3) implies (2) is trivial by taking $f(x)=v(x)^{-1 / p} \chi_{I}(x)$ and $\lambda=(1 / 2) \exp \left(\frac{1}{|I|} \int_{I} \log \left(v^{-1 / p}\right) d \mu\right)$.

For the proof that (2) implies (3) we need only consider the case $p=1$. As in the case of the proof of Theorem 1 we need only consider functions $f$ that are bounded with support of finite $\mu$ measure and $\int|f| v d \mu<\infty$ and weights $v$ with a positive lower bound. Given such functions $f$ and $v$ and a $\lambda>0$, define an operator $M_{f}$ by

$$
M_{f}(x)=\sup _{I} \frac{\int_{I}|f(y)| v(y) d \mu(y)}{\int_{I} w(t) d \mu(t)}
$$

with the sup taken over all intervals $I$ containing $x$ with the quotient taken as 0 in ambiguous cases. By (2), Jensen's inequality and the restrictions on $f$ and $v$

$$
\begin{aligned}
G_{f}(x) & =\sup _{I} \exp \left(\left[\frac{1}{|I|} \int_{I} \log (|f| v) d \mu\right]\left[\frac{1}{|I|} \int_{I} \log \left(\frac{1}{v}\right) d \mu\right]\right) \\
& \leq \sup _{I} C_{2} \frac{\frac{1}{|I|} \int_{I}|f| v d \mu}{\frac{1}{|I|} \int_{I} w d \mu}=C_{2} M_{f}(x) .
\end{aligned}
$$

Let $E$ be the set where $M_{f}(x)>\lambda / C_{2}$, let $D$ be the set where $M_{f}(x)=\infty$ and let $C$ be the collection of intervals $I$ such that $\int_{I}|f| v d \mu>0$ and $\int_{I} w d \mu=0$. Let $A$ be the sets of points $a$ such that $[a, a] \in C$. The assumption that $\int|f| v d \mu<\infty$ implies that $A$ is countable. The set $D \cap A^{c}$ is covered by intervals in $C$ with positive length. Therefore, by Lemma 2 and the countability of $A, D$ has a countable covering $\left\{I_{k}\right\}$ of intervals in $C$. From this $\int_{D} w d \mu \leq \sum_{k} \int_{I_{k}} w d \mu=0$. This and (9) show that

$$
\int_{\left\{G_{f}(x)>\lambda\right\}} w d \mu \leq \int_{E} w d \mu=\int_{E \cap D^{c}} w d \mu .
$$

Now let $B$ consist of all intervals $I$ with $\int_{I}|f| v d \mu>\frac{\lambda}{C_{2}} \int_{I} w d \mu>0$. Then $B$ covers $E \cap D^{c}$ and we can apply Lemma 1 with $d \nu=w d \mu$. The result is a disjoint 
sequence $\left\{J_{n}\right\}$ from $B$ and

$$
\int_{E \cap D^{c}} w d \mu \leq 5 \sum_{n} \int_{J_{n}} w d \mu<\frac{5 C_{2}}{\lambda} \sum_{n} \int_{J_{n}}|f| v d \mu \leq \frac{5 C_{2}}{\lambda} \int|f| v d \mu .
$$

Combining (10) and (11) completes the proof of Theorem 2.

\section{An example}

In this section we derive a pair $(w, v)$ of functions that satisfy (2) but do not satisfy (1). It is also easily seen that they do not satisfy (4).

Let $d \mu(x)=d x$,

$$
v(x)= \begin{cases}\exp \left[-\frac{1}{x(\log x)^{2}}\right], & x \in\left(0, e^{-2}\right] \\ \infty, & \text { elsewhere }\end{cases}
$$

and

$$
w(x)=\chi_{\left(0, e^{-2}\right]}(x) \frac{d}{d x}\left[x \exp \left(\frac{1}{x \log x}\right)\right] .
$$

Note that

$$
w(x)=\chi_{\left(0, e^{-2}\right]}(x)\left(1-\frac{1}{x \log x}-\frac{1}{x(\log x)^{2}}\right) \exp \left(\frac{1}{x \log x}\right)
$$

so that for $x$ in $\left(0, e^{-2}\right]$

$$
w(x) \leq \frac{-2}{x \log x} \exp \left(\frac{1}{x \log x}\right)
$$

and

$$
w(x) \geq \frac{-1}{2 x \log x} \exp \left(\frac{1}{x \log x}\right) .
$$

To prove (2) we need only consider intervals $I \subset\left[0, e^{-2}\right]$ since the second factor in (2) is 0 if $I \not \subset\left[0, e^{-2}\right]$. We will prove that (2) holds for $I=(a, b)$ in two cases: $a<2 b / 3$ and $a \geq 2 b / 3$.

For $a<2 b / 3$

$$
\frac{1}{|I|} \int_{I} w(x) d x \leq \frac{3}{b} \int_{0}^{b} w(x) d x=3 \exp \left(\frac{1}{b \log b}\right),
$$

and since $\log \left(\frac{1}{v}\right)=\frac{1}{x(\log x)^{2}}$ is decreasing on $\left(0, e^{-2}\right]$,

$$
\frac{1}{|I|} \int_{I} \log \left(\frac{1}{v(x)}\right) d x<\frac{1}{b} \int_{0}^{b} \log \left(\frac{1}{v(x)}\right) d x=\frac{-1}{b \log b} .
$$

Therefore,

$$
\exp \left[\frac{1}{|I|} \int_{I} \log \left(\frac{1}{v(x)}\right) d x\right]<\exp \left(\frac{-1}{b \log b}\right) .
$$

Combining (14) and (15) gives (2) with $C_{2}=3$ for this case.

For $a \geq 2 b / 3$ use (12) and the fact that the right side of (12) is increasing on $\left(0, e^{-2}\right]$ to see that

$$
\frac{1}{|I|} \int_{I} w(x) d x<\frac{-2}{b \log b} \exp \left(\frac{1}{b \log b}\right) .
$$


Next, since $\frac{1}{x(\log x)^{2}}$ decreases on $\left(0, e^{-2}\right]$,

$$
\frac{1}{|I|} \int_{I} \log \left(\frac{1}{v}\right) d x=\frac{1}{b-a} \int_{a}^{b} \frac{d x}{x(\log x)^{2}}<\frac{1}{a(\log a)^{2}}
$$

Now for $2 b / 3 \leq a<b \leq e^{-2}$

$$
\frac{1}{a(\log a)^{2}}<\frac{-1}{2 a \log a}<\frac{-1}{(4 / 3) b \log b}
$$

Using this, (16) and (17) gives

$$
\left[\frac{1}{|I|} \int_{I} w(x) d x\right]\left[\exp \left(\frac{1}{|I|} \int_{I} \log \left(\frac{1}{v(x)}\right) d x\right)\right]<\frac{-2}{b \log b} \exp \left(\frac{1}{4 b \log b}\right) .
$$

Since $8 y \exp (-y) \leq 8 / e$ for $y$ in $(0, \infty)$, the right side is bounded by $8 / e$. This completes the proof of (2) with $C_{2}=3$ for this pair.

To show that (1) is false for this $v$ and $w$ take $f(x)=v(x)^{-1 / p} \chi_{\left(0, e^{-2}\right)}(x)$. The integral on the right side of (1) is $e^{-2}$. Now

$$
G_{f}(x) \geq \exp \left(\frac{1}{x} \int_{0}^{x} \log f(t) d t\right)=\exp \left(\frac{-1}{p x \log x}\right) .
$$

This and (13) show that the left side of (1) is bounded below by $\int_{0}^{e^{-2}} \frac{-d x}{2 x \log x}=\infty$. This computation with $p=1$ also shows directly that (4) fails for this pair.

\section{REFERENCES}

1. N. Bourbaki, Elements de mathematique, Livre VI Integration, Hermann, Paris, 1952. MR 14: $960 \mathrm{~h}$

2. S. V. Hruscev, A description of weights satisfying the $A_{\infty}$ condition of, Muckenhoupt Proc. Amer. Math. Soc. 90 (1984), 253-257. MR 85k:42049

3. , W. Hu, X. Shi, and Q. Sun $A_{\infty}$ condition characterized by maximal geometric mean operator, Lecture Notes in Math., vol. 1494, Springer-Verlag, Berlin and New York, 1991, pp. 68-72. MR 94k:26023

4. B. Muckenhoupt, The equivalence of two conditions for weight functions, Studia Math. 49 (1974), 101-106. MR 50:2790

5. Weighted norm inequalities for the Hardy maximal function, Trans. Amer. Math. Soc. 165 (1972), 207-226. MR 45:2461

6. E. T. Sawyer, A characterization of a two-weight norm inequality for maximal, operators Studia Math. 75 (1982), 1-11. MR 84i:42032

7. X. Shi, Two inequalities related to geometric mean operators, J. Zhejiang Teacher's College 1 (1980), 21-25.

8. P. Sjogren, A remark on the maximal function for measures in $R^{n}$, Amer. J. Math. 105 (1983), 1231-1233. MR 86a:28003

Department of Mathematics, Hangzhou University, Zhejiang 310028, People's RepubLIC OF CHINA

Current address: Department of Mathematics and Statistics, McMaster University, Hamilton, Ontario, Canada L8S 4K1

E-mail address: xyin@icarus.math.mcmaster.ca

Department of Mathematics, Rutgers University, New Brunswick, New Jersey 08903 